\title{
Is there sign-tracking in aversive conditioning?
}

\author{
WILLIAM D. BARTTER and FRED A. MASTERSON \\ University of Delaware, Newark, Delaware 19711
}

\begin{abstract}
The present experiment studied the approach-withdrawal behavior of rats relative to conditioned stimuli (CS) that were paired with shock (paired), paired with absence of shock (unpaired), or that bore no temporal relation with shock (random). In the paired condition, the rats equally divided their time between the side of a tilt cage on which the CS was presented and the other side. In the random condition, the rats displayed a preference for the CS side. In the unpaired condition, the rats displayed a larger preference for the CS side. The range of preferences observed was small compared with that typically observed in appetitive sign-tracking. These results are consistent with a small aversive sign-tracking effect coupled with an unconditioned preference for the CS.
\end{abstract}

Hearst (1975) has emphasized the fact that the autoshaping paradigm (Brown \& Jenkins, 1968) appears to represent the classical conditioning of skeletal responses. These are coordinated, organized movements of the whole organism, in contrast to simple reflexive skeletal responses. Hearst and Jenkins (1974) have labeled this directed action sign-tracking.

An experiment by Wasserman, Franklin, and Hearst (1974) provides an example of sign-tracking. Four groups of pigeons were run in an operant chamber with a pivoted floor that allowed Wasserman et al. to monitor the pigeon's location. For the paired group, one of two side keys was illuminated immediately before each food presentation. The location of the illuminated key alternated randomly from trial to trial. For the unpaired group, food was presented during the intertrial interval, but never within $33 \mathrm{sec}$ of a key illumination. The results showed that the pigeons in the paired condition not only pecked the keys (the standard autoshaping finding), but also stood on the same side of the box that contained the illuminated key. More surprising, perhaps, was the performance of the pigeons in the unpaired condition. These birds spent most of their CS time on the side of the chamber opposite the lighted key. They withdrew from a cue signaling no food.

The purpose of the current experiment was to determine whether a sign-tracking analysis could be applied to aversive reinforcers, specifically, footshock. While the results of Biederman, D'Amato, and Keller (1964), McAdam (1964), and Whittleton, Kostanek, and Sawrey (1965) are consistent with aversive sign-tracking, the interpretation of these results is complicated by the existence of an instrumental avoidance contingency. In the absence of instrumental contingencies, will rats

William Bartter is now at Marietta College, Marietta, Ohio 45750. These experiments were reported at the 1977 convention of the Eastern Psychological Association in Boston. Requests for reprints should be addressed to Fred A. Masterson, Department of Psychology, University of Delaware, Newark, Delaware 19711. withdraw from a signal for shock and approach a signal for no shock?

\section{METHOD}

\section{Subjects}

Twenty-four male Wistar rats, approximately 90 days old, were used. They were maintained in individual cages with free access to food and water.

\section{Apparatus}

A $17 \mathrm{~cm}$ wide $\times 36 \mathrm{~cm}$ long $\mathrm{x} 19 \mathrm{~cm}$ high aluminum box was mounted over a pivoted grid floor. The fulcrum of the floor was in the center of the box, perpendicular to the longest walls. On each end wall a $2.5-\mathrm{cm}$ hole was drilled $5 \mathrm{~cm}$ above the grid floor. A standard keypeck manipulandum was positioned behind each hole. The onset of a light $(28 \mathrm{~V}$ dc delivered to a Sylvania 24 ESB bulb) behind each key served as the CS. Each CS presentation was $10 \mathrm{sec}$ long. The US was a 2 -sec $100-\mathrm{V}$ shock delivered by a $150 \mathrm{kohm}$ fixed-impedance ac shock source (Masterson \& Campbell, 1972), with the grid polarity pattern scrambled 4 times/sec by a stepping switch.

\section{Procedure}

All rats received $30 \mathrm{CS}$ presentations and 30 US presentations each day for 10 days. Each stimulus was presented on a variableinterval (VI) 2-min schedule. The location of the CS was randomly changed from side to side with the constraint that there be 15 trials on each side.

There were three groups in this experiment. Rats in the paired condition had each shock preceded by a 10-sec illumination of one of the key lights. For rats in the unpaired condition, key illuminations signaled a shock-free period of variable duration with a mean of $100 \mathrm{sec}$. There was also a variable interval between shock and the onset of the CS for the next trial. The mean duration of this interval was $20 \mathrm{sec}$, with a minimum of $15 \mathrm{sec}$. Finally, for rats in the random condition, there was no relation between the CSs and shocks. Shocks and signals were presented by two independent VI 2-min schedules.

\section{RESULTS}

Figure 1 presents the percent time that rats spent on the CS side of the box over the 10 days of the experiment. Scores at $50 \%$ represent indifference to the location of the signal; scores greater than $50 \%$ indicate approach responses; and scores less than $50 \%$ indicate 


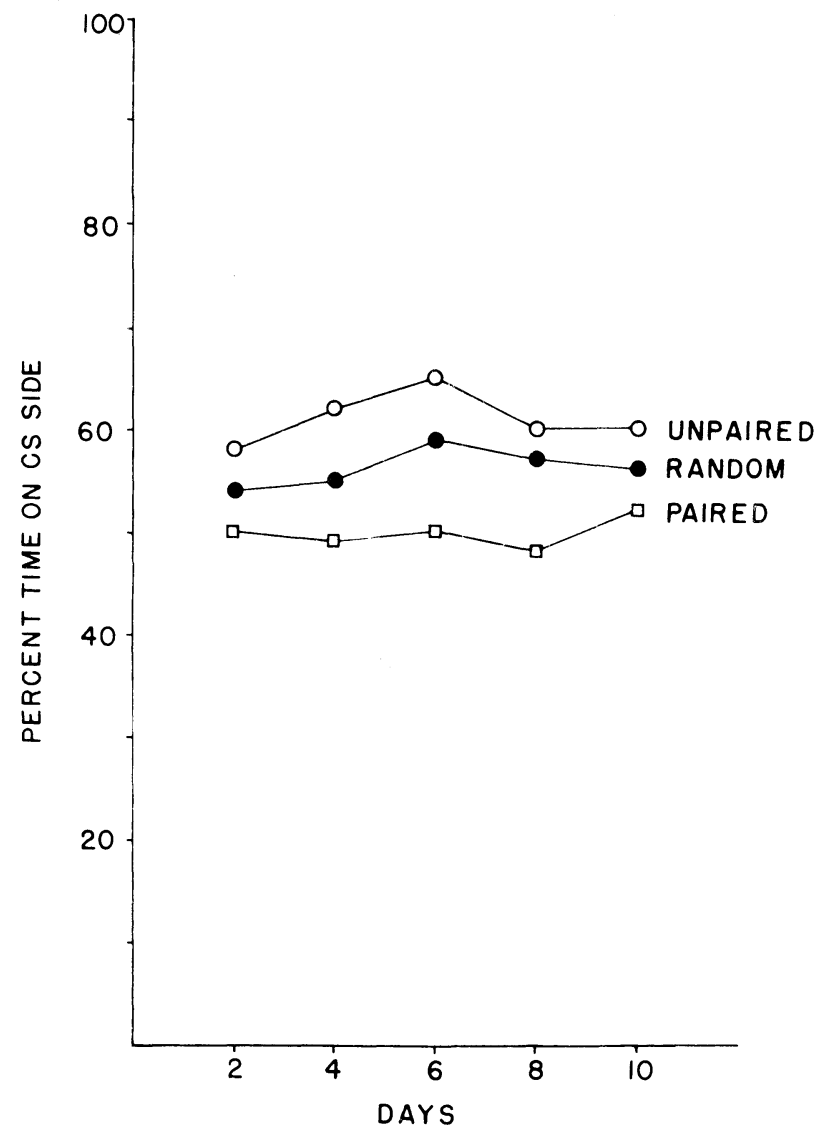

Figure 1. Mean percent CS time spent on the CS side of the choice apparatus.

withdrawal responses. It is clear that the rats in the paired condition show no consistent approach or withdrawal behaviors. On the other hand, rats in both the unpaired and random conditions show an initial tendency to spend more time on the signal side. This tendency increases over the first 6 days and then decreases over the final 4 days.

An analysis of variance showed a significant main effect $[F(2,21)=9.9, p<.01]$. A subsequent NewmanKeuls test revealed that this effect resulted from a difference between the unpaired and paired groups $(p<.01)$ and a difference between the random and paired groups $(p<.05)$. There were no significant trials effects or interaction effects.

The number of times the animals changed sides was recorded both during the signals and during the intertrial intervals (ITIs). The only significant effect was that, overall, the rats changed sides more frequently during signal periods than during ITI periods $[\mathrm{F}(1,21)=6.3$, $p<.05]$. There was no significant interaction effect.

We also analyzed the number of contacts the rats made with the keys. There was a significant main effect $[F(2,21)=4.2, p<.05]$. A Newman-Keuls test showed that the rats in the paired condition pressed the keys less frequently than the rats in either the unpaired or the random condition (both ps $<.05$ ). There was also a significant periods effect $[\mathrm{F}(1,21)=13.6, \mathrm{p}<.01]$, indicating that there were more contacts when the signals were on than when they were off. There was a significant Groups by Periods interaction $[F(2,21)=6.4$, $\mathrm{p}<.01$ ], which resulted from the fact that the unpaired rats pressed the keys more during the signal periods than did any other group at any other time (Newman-Keuls test, $\mathrm{p}<.01)$.

\section{DISCUSSION}

The relative orderings of the preferences provide some evidence for sign-tracking. Both the unpaired and the random conditions produced stronger preferences for the CS side than did the paired condition. Furthermore, while not statistically significant, the rats in the unpaired group spent more time on the CS side than did rats in the random group. However, the absolute preferences are not consistent with an explanation based entirely on sign-tracking. According to such an explanation, the random condition should produce indifference to the CS, yet our random group showed a slight preference for the CS side. Also, the paired condition should prefer the non-CS side, yet our paired group spent equal amounts of time on either side.

The results could be interpreted as due to an aversive signtracking effect superimposed on an unconditioned preference for the CS side over the non-CS side. Such a preference might be due to investigative responses directed toward the CS. For example, Masterson (Note 1) measured a high incidence (185 per 1-h session) of unconditioned nose-poking responses directed toward an illuminated key in the absence of shock. It is possible that a similar investigative response tendency was operating in the current experiment.

To test this interpretation, eight rats, similar to those run in the above experiment, were run in the same apparatus for 10 daily sessions. The procedure was the same except these rats were never shocked. The rats showed a statistically reliable $58 \%$ preference for the side with the lighted key during the first 2 days, suggesting an unconditioned attraction to the lighted key. This preference decreased to $54 \%$ over the remaining 8 days. A plausible explanation of this decrease is that the rats became inactive when the novelty of the apparatus wore off, and they may even have fallen asleep. By contrast, the rats in the random group of the current experiment probably remained active and alert, since they were periodically shocked. As a result, their preference for the lighted key continued to reflect an unabated unconditioned attraction for the lighted key over the entire 10-day experiment.

The approach and withdrawal effects observed in the current aversive sign-tracking paradigm are weaker than those observed in appetitive sign-tracking. A possible explanation is that we used a faulty analogy between the appetitive and aversive situations. If the key to appetitive sign-tracking is the relationship of the CS with an appetitive UCS, then, by analogy, one might expect the basis for aversive sign-tracking to be the relationship between the CS and an aversive UCS. Our present results suggest that this analogy is incorrect. A better analogy might involve the notion of positive incentive stimuli. For the case of hunger motivation, food is an unconditioned positive incentive stimulus. Elsewhere, we have presented a consummatory stimulus theory of the rat's flight behavior (Masterson, Crawford, \& Bartter, 1978) that assumes that flight from an aversive place generates rewarding consummatory stimuli. Pursuing this new analogy, positive sign-tracking would involve approaching a CS that is paired with an opportunity to flee a dangerous place, whereas negative signtracking would involve withdrawing from a CS that is followed by a period of time devoid of such an opportunity. 
One of the most important sources of positive incentive may be the stimulus changes generated by getting away from the shock grids (Testa, 1974). For example, Moderesi (1975) found that rats learn shuttle avoidance as fast as one-way avoidance when retractable shelves are used to cover the grids on the currently safe side of the shuttlebox. Pursuing the positive incentive analogy for aversive sign-tracking, we propose a paradigm in which CSs are related, not to shock events, but to appearances of a retractable shelf upon which the animals can hop. According to the new analogy, rats should acquire approach responses toward a CS that predicts the arrival of the shelf, and they should acquire withdrawal responses from a CS that predicts that the shelf will not appear in the immediate future. This analogy may lead to aversive sign-tracking effects comparable to those observed in the appetitive case.

\section{REFERENCE NOTE}

1. Masterson, F. W. Unpublished data, 1963.

\section{REFERENCES}

Biederman, G. B., D'Amato, M. R., \& Keller, D. M. Facilitation of discriminated avoidance learning by dissociation of CS and manipulandum. Psychonomic Science, 1964, 1, 229-230.

Brown, P. L., \& Jenkins, H. M. Auto-shaping of the pigeon's key-peck. Journal of the Experimental Analysis of Behavior, $1968,11,1-8$

Hearst, E. The classical-instrumental distinction: Reflexes, voluntary behavior, and categories of associative learning. In W. K. Estes (Ed.), Handbook of learning and cognitive processes (Vol. 2). Hillsdale, N.J: Erlbaum, 1975.

HeArSt, E., \& Jenkins, H. M. Sign-tracking: The stimulusreinforcer relation and directed action. Austin, Tex: Psychonomic Society, 1974.

Masterson, F. A., \& Campbell, B. A. Techniques of electric shock motivation. In R. D. Myers (Ed.), Methods in psychology: Laboratory techniques in neuropsychology. New York: Academic Press, 1972.

Masterson, F. A., Crawford, M., \& Bartter, W. D. Brief escape from a dangerous place: The role of reinforcement in the rat's one way avoidance acquisition. Learning and Motivation, 1978, 9, 141-163.

McАдам, D. Effects of positional relations between subjects, CS, and US on shuttle-box avoidance learning in cats. Journal of Comparative and Physiological Psychology, 1964, 58, 302-304.

Modaresi, H. One-way characteristic performance of rats under two-way signaled avoidance conditions. Learning and Motivation, 1975, 6, 484-497.

Testa, T. J. Causal relationships and the acquisition of avoidance responses. Psychological Review, 1974, 81, 491-505.

Wasserman, E. A., Franklin, S. R., \& Hearst, E. Pavlovian appetitive contingencies and approach versus withdrawal to conditioned stimuli in pigeons. Journal of Comparative and Physiological Psychology, 1974, 86, 616-627.

Whittleton, J. C., Kostanek, D. J., \& Sawrey, J. M. CS directionality and intensity in avoidance learning and extinction. Psychonomic Science, 1965, 3, 415-416.

(Received for publication November 15, 1979.)

\section{ERRATUM}

Leitner, D. S., Powers, A. S., \& Hoffman, H. S. The neural system for the inhibition of startle. Bulletin of the Psychonomic Society, 1979, 14 (6), 410-412. The first line of the Results section should read: In both animals with lesions of the lateral tegmentum, two structures were severely damaged bilaterally: the posterior part of the nucleus cuneiformis, including Cell Group A8 of Dahlstrom and Fuxe (1964), and the nucleus parabrachialis ventralis. 